\title{
Lattice Boltzmann and Gas Kinetic Flux Solvers and Their Applications
}

\author{
Chang Shu \\ Department of Mechanical Engineering \\ National University of Singapore, Singapore
}

\begin{abstract}
The macroscopic governing differential equations in fluid mechanics can be discretized by the finite volume method (FVM), and the conservative flow variables at cell centres can be given from the solution of discrete governing equations. In the solution process, we need to evaluate numerical fluxes at cell interfaces from the solution at cell centres. Currently, there are two kinds of approaches to reconstruct the solution for evaluation of numerical fluxes. One is mathematical approach, which is based on smooth function approximation. The other is physical approach, which is based on the solution of governing equation or simplified governing equation. A good example in this category is the Riemann solver, which is based on solution of one-dimensional Euler equation. In this talk, a brief review of current flux solvers will be presented first. Then the newly-developed lattice Boltzmann flux solver and gas kinetic flux solver will be shown in details. These solvers evaluate viscous and inviscid fluxes simultaneously and physically. They combine advantages of conventional Navier-Stokes solvers and lattice Boltzmann/gas kinetic solvers. The fluid flows from incompressible regime to hypersonic regime can be well simulated by these solvers. The proposed new flux solvers will be validated by various numerical examples.
\end{abstract}

